Table A. Transitions between depression symptoms at baseline and depression symptoms or a cardiovascular event at 5 and 10 years by age group with gender, body mass index, smoking, education, diabetes, myocardial infarction, physical activity and anti-depression/anxiety medication; transition probabilities are shown at the reference level, $N=997$.

\begin{tabular}{|c|c|c|c|c|c|c|c|c|c|}
\hline \multirow{2}{*}{$\begin{array}{l}\text { Level of } \\
\text { depression } \\
\text { symptoms at } \\
\text { baseline by age } \\
\text { groups }\end{array}$} & \multirow[b]{2}{*}{$\mathrm{N}$} & \multicolumn{4}{|c|}{ Transition probabilities (\%) at 5 years } & \multicolumn{4}{|c|}{ Transition probabilities (\%) at 10 years } \\
\hline & & $\begin{array}{c}\text { No } \\
\text { symptoms } \\
(<8)\end{array}$ & $\begin{array}{l}\text { Mild } \\
(8-10)\end{array}$ & $\begin{array}{c}\text { Moderate/severe } \\
(\geq 11)\end{array}$ & CVE & $\begin{array}{c}\text { No } \\
\text { symptoms } \\
(<8)\end{array}$ & $\begin{array}{l}\text { Mild } \\
(8-10)\end{array}$ & $\begin{array}{c}\text { Moderate/severe } \\
(\geq 11)\end{array}$ & CVE \\
\hline \multicolumn{10}{|c|}{ No symptoms $(<8)$} \\
\hline$<55 \mathrm{yr}$ & 203 & 89.2 & 5.3 & 0.8 & 4.6 & 83.7 & 3.8 & 1.0 & 11.5 \\
\hline $55-64 \mathrm{yr}$ & 383 & 86.9 & 5.4 & 1.0 & 6.7 & 78.2 & 4.1 & 1.1 & 16.5 \\
\hline$>64 \mathrm{yr}$ & 241 & 83.9 & 5.3 & 0.9 & 9.8 & 71.2 & 4.3 & 0.8 & 23.7 \\
\hline \multicolumn{10}{|l|}{ Mild (8-10) } \\
\hline$<55 \mathrm{yr}$ & 26 & 63.0 & 27.4 & 5.8 & 3.8 & 79.1 & 7.3 & 2.7 & 10.8 \\
\hline $55-64 \mathrm{yr}$ & 58 & 53.4 & 33.1 & 7.7 & 5.9 & 69.7 & 10.1 & 3.4 & 16.9 \\
\hline$>64 \mathrm{yr}$ & 30 & 44.0 & 37.6 & 8.2 & 10.3 & 57.6 & 12.4 & 2.8 & 27.3 \\
\hline \multicolumn{10}{|l|}{$\begin{array}{l}\text { Moderate/severe } \\
(\geq 11)\end{array}$} \\
\hline$<55 \mathrm{yr}$ & 21 & 48.4 & 24.3 & 22.4 & 4.8 & 72.8 & 8.6 & 5.7 & 12.9 \\
\hline $55-64 \mathrm{yr}$ & 24 & 42.8 & 21.9 & 25.7 & 9.6 & 61.6 & 9.3 & 6.0 & 23.1 \\
\hline$>64 \mathrm{yr}$ & 11 & 37.7 & 18.5 & 24.0 & 19.8 & 48.0 & 8.2 & 3.5 & 40.3 \\
\hline
\end{tabular}

CVE: cardiovascular event; shaded area show same level of symptoms at baseline and during follow-up 
Table B. Transitions between severity of anxiety symptoms at baseline and anxiety symptoms or a cardiovascular event at 5 and 10 years by age group with gender, body mass index, smoking, education, diabetes, myocardial infarction, physical activity and anti-depression/anxiety medication; transition probabilities are shown at the reference level, $\mathrm{N}=$ 997.

\begin{tabular}{|c|c|c|c|c|c|c|c|c|c|}
\hline \multirow{2}{*}{$\begin{array}{l}\text { Level of anxiety } \\
\text { symptoms at } \\
\text { baseline by age } \\
\text { groups }\end{array}$} & \multirow[b]{2}{*}{$\mathbf{N}$} & \multicolumn{4}{|c|}{ Probabilities (\%) at 5 years } & \multicolumn{4}{|c|}{ Probabilities (\%) at 10 years } \\
\hline & & $\begin{array}{c}\text { No } \\
\text { symptoms } \\
(<8) \\
\end{array}$ & $\begin{array}{l}\text { Mild } \\
(8-10)\end{array}$ & $\begin{array}{c}\text { Moderate/severe } \\
(\geq 11)\end{array}$ & CVE & $\begin{array}{c}\text { No } \\
\text { symptoms } \\
(<8) \\
\end{array}$ & $\begin{array}{l}\text { Mild } \\
(8-10)\end{array}$ & $\begin{array}{c}\text { Moderate/severe } \\
(\geq 11)\end{array}$ & CVE \\
\hline \multicolumn{10}{|c|}{ No symptoms $(<8)$} \\
\hline$<55 \mathrm{yr}$ & 173 & 86.5 & 7.2 & 1.7 & 4.6 & 80.0 & 6.4 & 1.9 & 11.8 \\
\hline $55-64 \mathrm{yr}$ & 361 & 85.8 & 5.9 & 1.4 & 6.9 & 76.0 & 5.2 & 1.3 & 17.5 \\
\hline$>64 \mathrm{yr}$ & 231 & 83.9 & 4.7 & 1.0 & 10.4 & 69.8 & 3.9 & 0.7 & 25.6 \\
\hline \multicolumn{10}{|l|}{ Mild (8-10) } \\
\hline$<55 \mathrm{yr}$ & 46 & 53.9 & 32.5 & 10.1 & 3.5 & 69.5 & 14.2 & 5.6 & 10.7 \\
\hline $55-64 \mathrm{yr}$ & 75 & 50.1 & 33.4 & 10.7 & 5.8 & 63.9 & 14.3 & 4.7 & 17.1 \\
\hline$>64 \mathrm{yr}$ & 36 & 45.7 & 33.8 & 10.6 & 9.8 & 56.0 & 13.7 & 3.1 & 27.2 \\
\hline \multicolumn{10}{|l|}{$\begin{array}{l}\text { Moderate/severe } \\
(\geq 11)\end{array}$} \\
\hline$<55 \mathrm{yr}$ & 31 & 32.9 & 22.8 & 40.3 & 4.1 & 58.0 & 17.1 & 12.6 & 12.3 \\
\hline $55-64 \mathrm{yr}$ & 29 & 32.2 & 24.9 & 35.4 & 7.5 & 53.7 & 16.9 & 8.8 & 20.6 \\
\hline$>64 \mathrm{yr}$ & 15 & 30.8 & 26.5 & 29.0 & 13.8 & 46.8 & 15.3 & 4.6 & 33.3 \\
\hline
\end{tabular}

CVE: cardiovascular event; shaded area show same level of symptoms at baseline and during follow-up 
Table C. Transitions between depression symptoms at baseline or a cardiovascular event at 5 and 10 years by physical activity level at one year with gender, body mass index, smoking, education, diabetes, history of myocardial infarction, physical activity and anti-depression/anxiety medication; transition probabilities are shown at the reference level, $\mathrm{N}=997$.

Level of depression symptoms at baseline

No symptoms $(<8)$

PA level 1, daily or 56x/week

PA level 2, 2-4x/week

PA level 3, 1x/week or $1-$

$3 x /$ month

PA level 4, seldom or

never

\section{Mild (8-10)}

PA level 1 , daily or 5 $6 x /$ week

PA level 2, 2-4x/week

PA level 3, 1x/week or 1$3 x /$ month

PA level 4 , seldom or never

Moderate/severe $(\geq 11)$

PA level 1 , daily or $5-$

$6 x /$ week

PA level 2, 2-4x/week

PA level $3,1 \mathrm{x} /$ week or 1 -

$3 x /$ month

PA level 4 , seldom or never by physical activity level

Transition probabilities (\%) at 5 years

\begin{tabular}{|c|c|c|c|}
\hline $\begin{array}{c}\text { No } \\
\text { symptoms } \\
(<8)\end{array}$ & $\begin{array}{l}\text { Mild } \\
(8-10)\end{array}$ & $\begin{array}{c}\text { Moderate/ } \\
\text { severe }(\geq 11)\end{array}$ & CVE \\
\hline
\end{tabular}
Transition probabilities (\%) at 10 years

\begin{tabular}{|c|c|c|c|c|c|c|c|c|}
\hline & $(<8)$ & $(8-10)$ & severe $(\geq 11)$ & & $(<8)$ & $(8-10)$ & severe $(\geq 11)$ & \\
\hline 276 & 89.2 & 5.3 & 0.8 & 4.6 & 83.7 & 3.8 & 1.0 & 11.5 \\
\hline 351 & 86.4 & 6.9 & 1.5 & 5.2 & 79.7 & 5.3 & 1.9 & 13.1 \\
\hline 134 & 82.7 & 8.8 & 2.6 & 5.9 & 74.3 & 7.2 & 3.1 & 15.4 \\
\hline 63 & 77.9 & 11.0 & 4.1 & 7.1 & 67.2 & 9.1 & 4.4 & 19.3 \\
\hline 25 & 63.0 & 27.4 & 5.8 & 3.8 & 79.1 & 7.3 & 2.7 & 10.8 \\
\hline 42 & 54.1 & 32.4 & 9.1 & 4.5 & 71.3 & 10.5 & 5.0 & 13.2 \\
\hline 32 & 45.2 & 36.3 & 12.7 & 5.8 & 61.4 & 13.5 & 7.4 & 17.6 \\
\hline 15 & 36.8 & 38.8 & 16.1 & 8.4 & 50.2 & 15.8 & 9.1 & 25.0 \\
\hline 9 & 48.4 & 24.3 & 22.4 & 4.8 & 72.8 & 8.6 & 5.7 & 12.9 \\
\hline 24 & 38.4 & 23.3 & 31.4 & 7.0 & 61.1 & 10.8 & 9.8 & 18.2 \\
\hline 14 & 29.5 & 20.7 & 39.0 & 10.7 & 47.9 & 11.6 & 13.1 & 27.4 \\
\hline 9 & 22.1 & 17.5 & 43.7 & 16.7 & 34.8 & 10.8 & 13.7 & 40.6 \\
\hline
\end{tabular}

cardiovascular event; PA: physical activity measured at one year; shaded area show same level of symptoms at baseline and during follow-up

$\begin{array}{cccc}\begin{array}{c}\text { No } \\ \text { symptoms } \\ (<8)\end{array} & \begin{array}{c}\text { Mild } \\ (8-10)\end{array} & \begin{array}{c}\text { Moderate/ } \\ \text { severe }(\geq 11)\end{array} & \text { CVE }\end{array}$


Table D. Transitions between anxiety symptoms at baseline or a cardiovascular event at 5 and 10 years by physical activity level at one year by age group with gender, body mass index, smoking, education, diabetes, history of myocardial infarction, and anti-depression/anxiety medication; transition probabilities are shown at the reference level, $\mathbf{N}=997$.

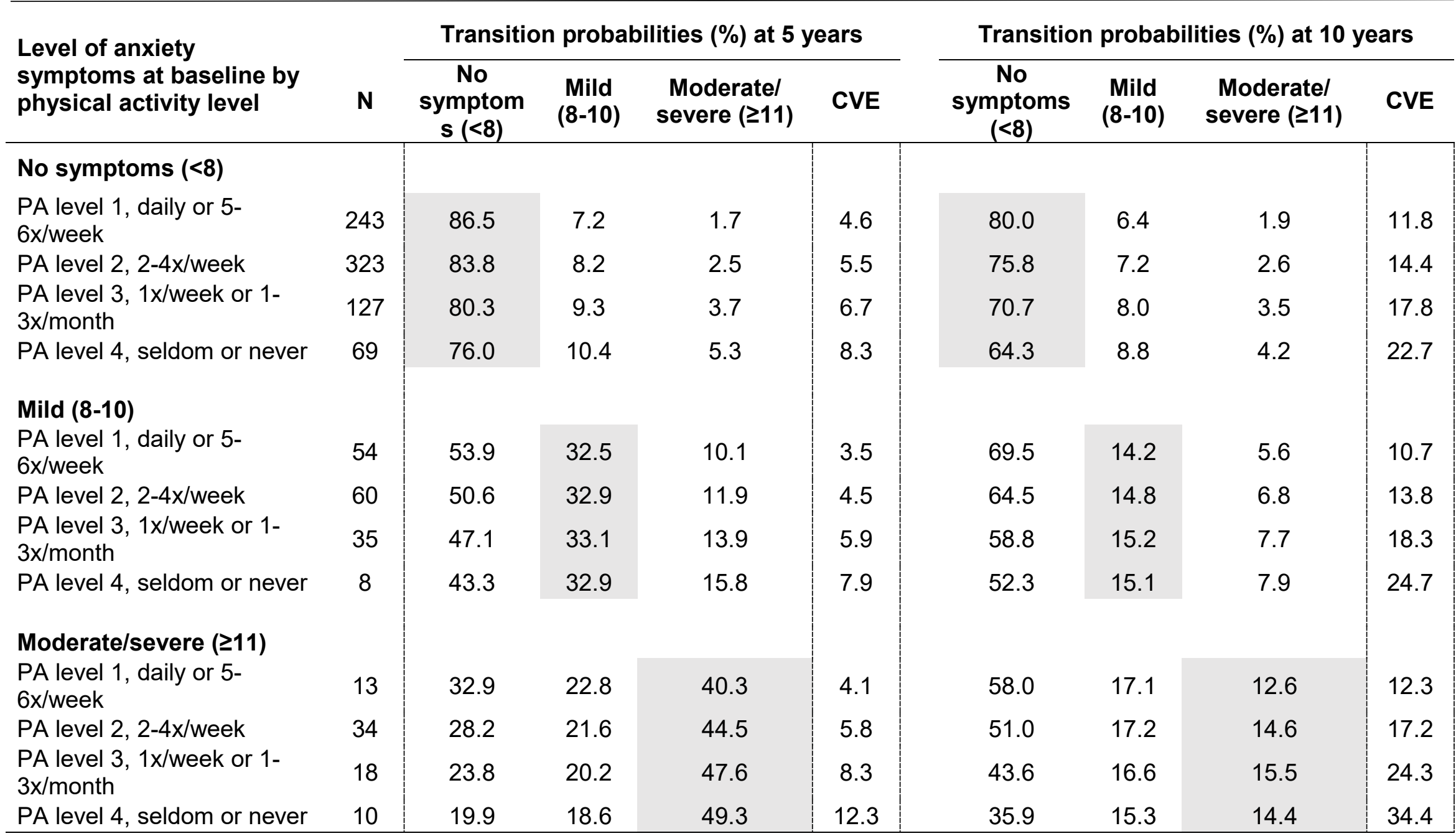

CVE: cardiovascular event; PA: physical activity measured at one year; shaded area show same level of symptoms at baseline and during follow-up 